\title{
Development of SCR VIV in a changing flow field
}

\author{
J. M. Niedzwecki ${ }^{1}$ \& G. $\mathrm{Moe}^{2}$ \\ ${ }^{I}$ Department of Civil Engineering, \\ Texas A\&M University College Station, Texas, USA \\ ${ }^{2}$ Department of Civil Engineering, NTNU, Trondheim, Norway
}

\begin{abstract}
The vortex induced vibrations targeted in laboratory model testing programs or the vortex induced motions observed in many offshore systems remain a subject of considerable interest and practical importance. In this study the role of a varying flow field observed in the towing of a steel catenary riser model on the analysis of the measured flow induced vibration phenomena is investigated. More specifically, in this study the variation of the current flow field that begins at or near zero and increases to a plateau level for the majority of the test run before tapering off to near zero flow conditions is analyzed. A transformation of the bi-axial data from Cartesian to Polar coordinates is used as a basis for interpreting the measurements. By investigating the response of the SCR model during the flow transition regions together with the region where the response behaviour appears to be somewhat stabilized, one may gain some insight into this complex response phenomena and the implications of truncating time series records in the process of analyses.

Keywords: SCR, catenary riser, model test, Polar coordinate system, autospectral analysis, Weibull distribution.
\end{abstract}

\section{Introduction}

As a whole, the offshore community has directed a great deal of attention upon developing a better understanding of vortex induced vibrations (VIV) of slender bodies and the vortex induced motions (VIM) of deepwater platforms [1-4]. Steel catenary risers (SCRs) have become an increasingly important component in the operation of an increasing number of offshore activities. Consequently, 
interested has increased in study of their hydrodynamic response behaviour especially in flow conditions that result in vortex induced vibrations (VIV). In this study data was selected from a laboratory study where a steel catenary riser model was towed at various speeds and angular orientations with respect to the towing direction $[1,2]$. Towing of the SCR model commences when the fluid in the tow tank has reached an acceptable quiescent state and the model is hanging in its static catenary configuration. The towing carriage then accelerates to reach the desired towing velocity, at which point the fluid flow field has a nearly uniform profile. Towing of the SCR model at a constant towing speed continues until the towing carriage reaches a point where it must decelerate and eventually stop. The effect of the ramping of the towing speeds on the interpretation of the SCR response behaviour is investigated. In particular, a comparison of the complete time series records with the same time series truncated in an attempt to isolate steady-state response behaviour are studied.

This study builds upon an earlier study research investigation of the SCR model test data [5], but approaches the analysis from an entirely different perspective. Here the bi-directional time series response measurements are transformed into an equivalent polar coordinate representation, and that leads to an interesting perspective from which to view the combined in-line and crossflow response of flexible marine systems. Further, the time series measurements of the towing speed and the displacement time records are used to guide the interpretation of the transition regions. This information is then used in the subsequent truncation of the measured time series. The objective is not to provide a predictive model but rather to develop a better understanding of the VIV response measurements through the use of a coordinate transformation, and statistical and probabilistic techniques.

\section{Experiment particulars}

The angular orientations and the location of equally-spaced bi-axial accelerometers, depicted by the circles along the static catenary curves, are illustrated in Fig 1. Ten bi-axial accelerometers were affixed to the SCR model and they are sequentially numbered starting from the lower end of the catenary curve. In this study, the towing carriage speed will be used along with the biaxial displacement information processed form the acceleration measurements. The three force components were measured at both of the fixed ends is not utilized in this study.

The model tests were conducted in a towing tank at Marintek [1, 2]. The towing tank used was $80 \mathrm{~m}$ long, $10.5 \mathrm{~m}$ wide and $10 \mathrm{~m}$ deep. The SCR model was $12.5 \mathrm{~m}$ long and had a diameter of $14 \mathrm{~mm}$. The ratio of the horizontal to vertical projection was approximately 1.3 which is consistent with the schematic presented in Fig. 1. The model was towed at speeds ranging from $0.12-0.26 \mathrm{~m} / \mathrm{s}$ at orientations of $0,30,60$ and 90 degrees. In this research study only the velocity the data associated with $0.26 \mathrm{~m} / \mathrm{s}$ and an orientation of 90 degrees is investigated. 


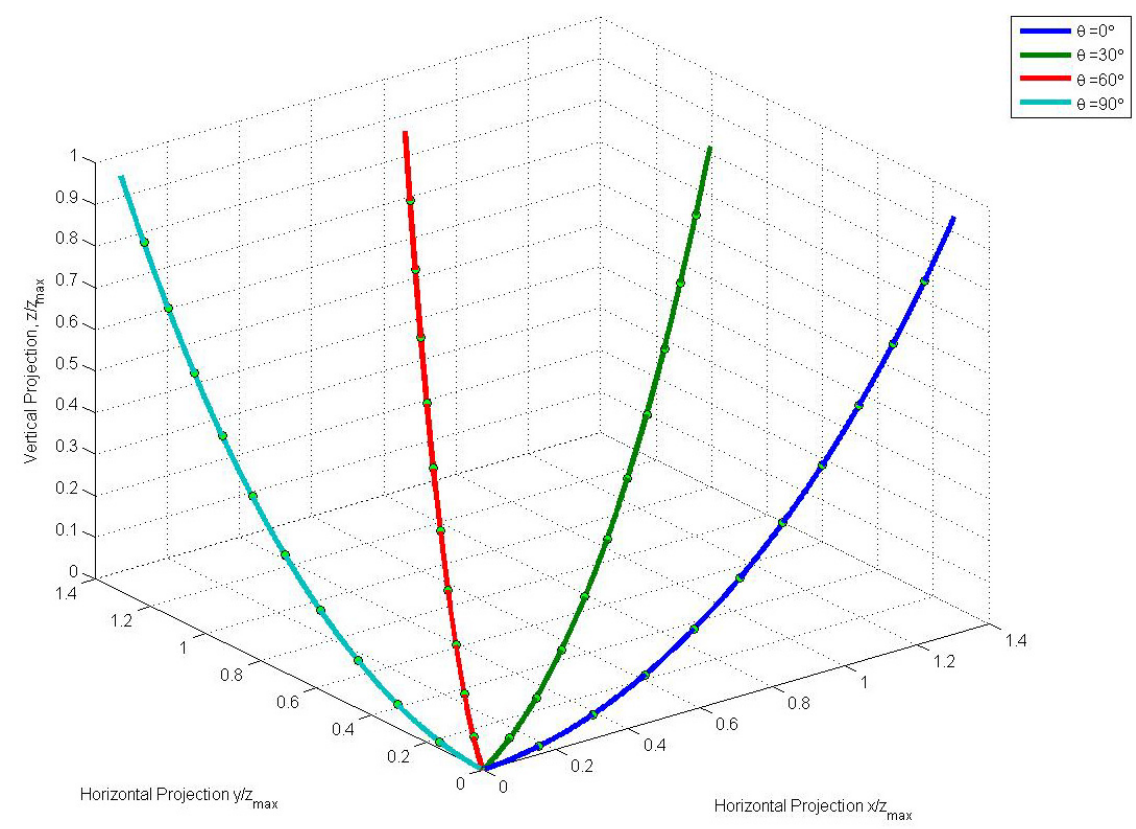

Figure 1: Schematic of the catenary model orientations and bi-axial accelerometers.

\section{Analysis of the data}

The analysis of the SCR data is for $\theta=90^{\circ}$ at a towing speed of $0.26 \mathrm{~m} / \mathrm{s}$. Referring to Fig. 1, the SCR is being towed in the negative y direction and the resulting SCR cross-flow motion occurs in the $\mathrm{x}-\mathrm{y}$ plane at the elevation of the bi-axial accelerometers, denoted by the circular marking along the catenary.

\subsection{Time series from the experiment}

A sample of the original data and the truncated data at the middle of the SCR model, bi-axial accelerometer \#5, is shown in Fig.2. Presented in that figure are the dimensionless components of the horizontal displacements, $x / d o$ and $y / d o$, and the carriage speed normalized by it maximum speed. In Fig. 2(a), the transient motion at the beginning of the test run and the slowing down of the towing carriage speed is evident. Even taking into account the ramp-up of the towing carriage velocity, it takes a bit longer for the displacement time series to approach a regular oscillatory pattern. As the towing carriage speed ramps down the VIV motions takes a while longer to decrease. The original data contained over 14,000 data points and based upon these considerations the truncated data sets have been reduced, Fig. 2(b), to just over 8,000 data points. This is a significant reduction and the logical question is what impact does truncating the 
time series records have on the analysis and conclusions regarding the response behaviour? To investigate this further, both the complete time series and the truncated time series will be compared.
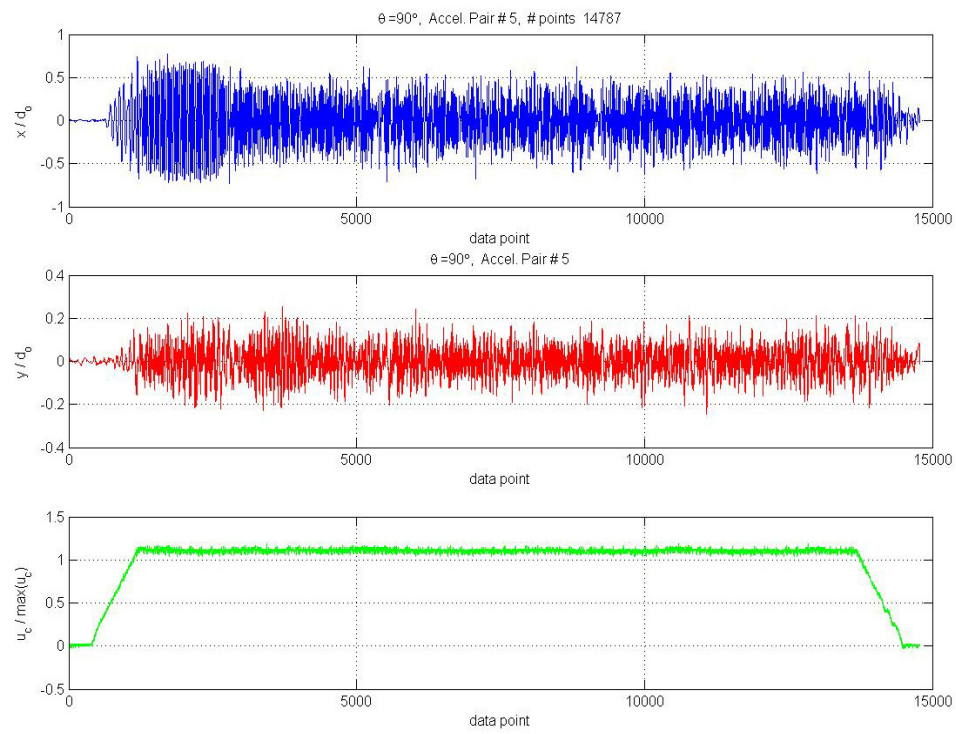

(a)
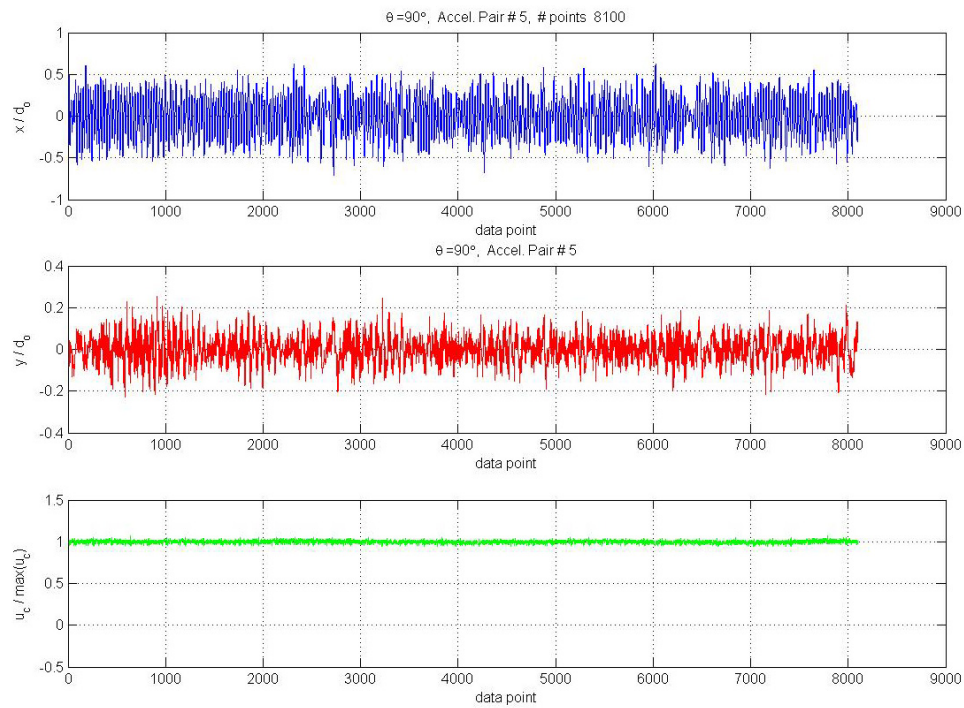

(b)

Figure 2: $\quad$ Time series of the component displacements from measurements at biaxial acceleration pair \#5 and the normalized towing speed (a) complete measurement and, (b) the truncated records. 


\subsection{Distributions at selected elevations}

Rather than working with the displacement measurements in Cartesian coordinates, $(x, y, z)$ it was decided to transform the data to Polar coordinates, $(r, \phi)$ at each of the measurement elevations. As a consequence of this transformation focus is directed at the amplitude for the response behaviour as illustrated in Fig. 3. The polar plot of the response is shown to the left in both Fig. 3(a) and Fig. 3(b). The corresponding distribution of the radial and angular distributions is shown next to the polar plots. Comparing the polar plots for the complete measurement records with the truncated records at each the top, middle and lower bi-axial accelerometer elevations suggest that the radial amplitudes are slightly smaller and the angular information is a bit more concentrated. At the middle elevation the motions of the complete record has more curvature in the polar plot and suggests that a closer look at the nature of the extremes is needed. In Fig. 4, Weibull probability plots of the radial components at the various measurement elevations are presented. For the complete measurement records there is a fairly large deviation at the smaller radial values, whereas the behaviour of truncated measurement records is reasonably well behaved and the standard deviation of the shape and scale parameters in the Weibull model are significantly reduced.

Reflecting upon these results the variation of the sorted radial component was envisioned and the results are presented in Fig. 5. When the data is viewed from this perspective the complete data records shows a separation of the data at several elevations, whereas when the truncated data is plotted it seems to behave in a more regular fashion. Another feature which was removed by the truncation was the difference in the radial component at the smallest values. This suggests that the effects of truncating the data measurements may be initially assessed with this representation of the data.

\subsection{Auto-spectral density at selected elevations}

The multi-model response of the SCR model is confirmed by the spectral plots presented in Fig. 6 and Fig. 7. In each figure the cross-flow spectrum $G x x(\omega)$ and the in-line flow spectrum $G y y(\omega)$ are depicted. Here the use of the terminology in-line refers to the direction in which the model is towed and cross-flow refers to the direction of vortex induced vibrations. The peaks of the cross-flow spectrum correspond quite well to frequency estimates made using predictions that combine axial and bending stiffness contributions. Further, the peaks of the cross-flow and in-line spectra are different and do not contain nearly the intensity of the VIV. 
90 Fluid Structure Interaction and Moving Boundary Problems IV
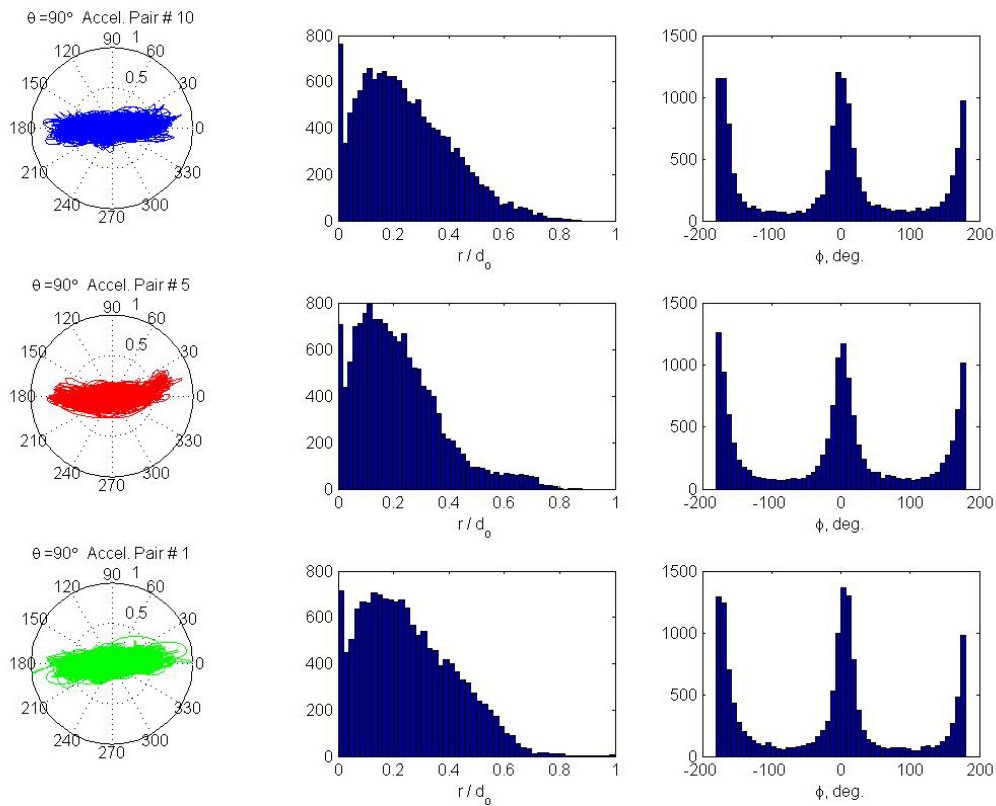

(a)
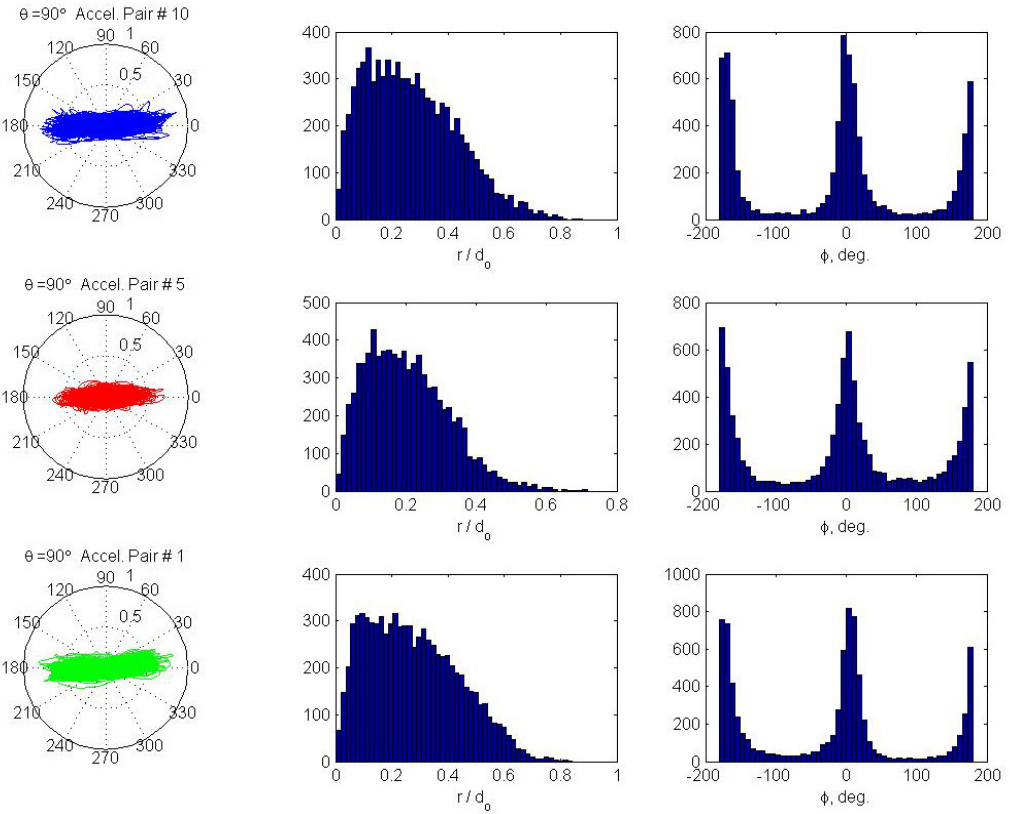

(b)

Figure 3: Comparison of the displacements at the top, bottom and middle of the SCR model (a) complete measurement and, (b) the truncated records. 


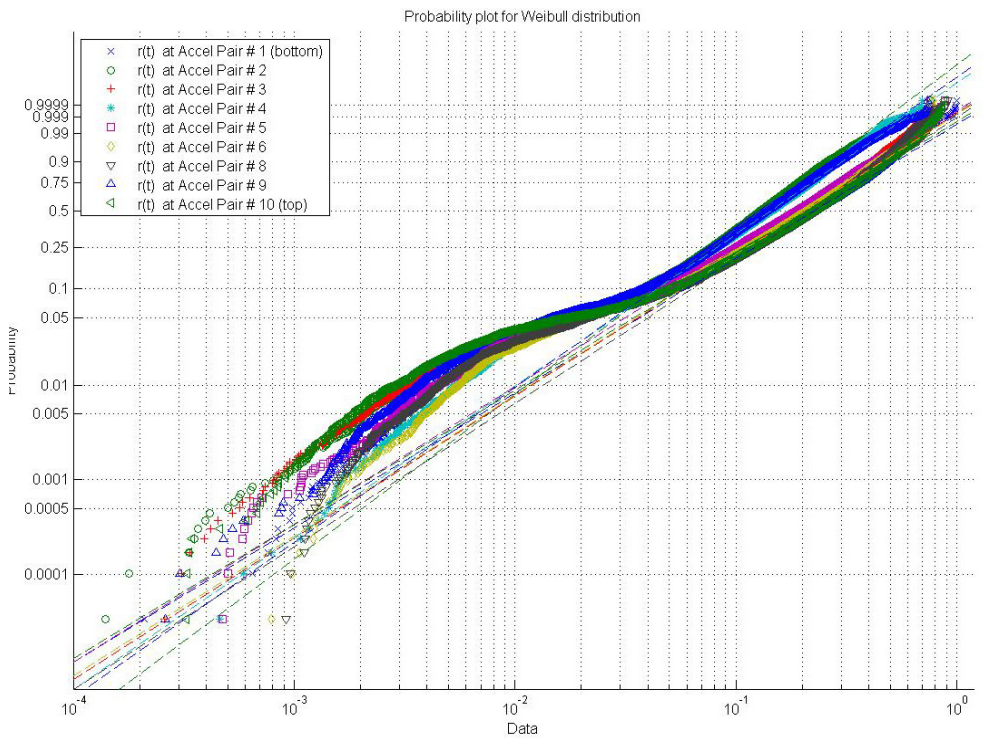

(a)

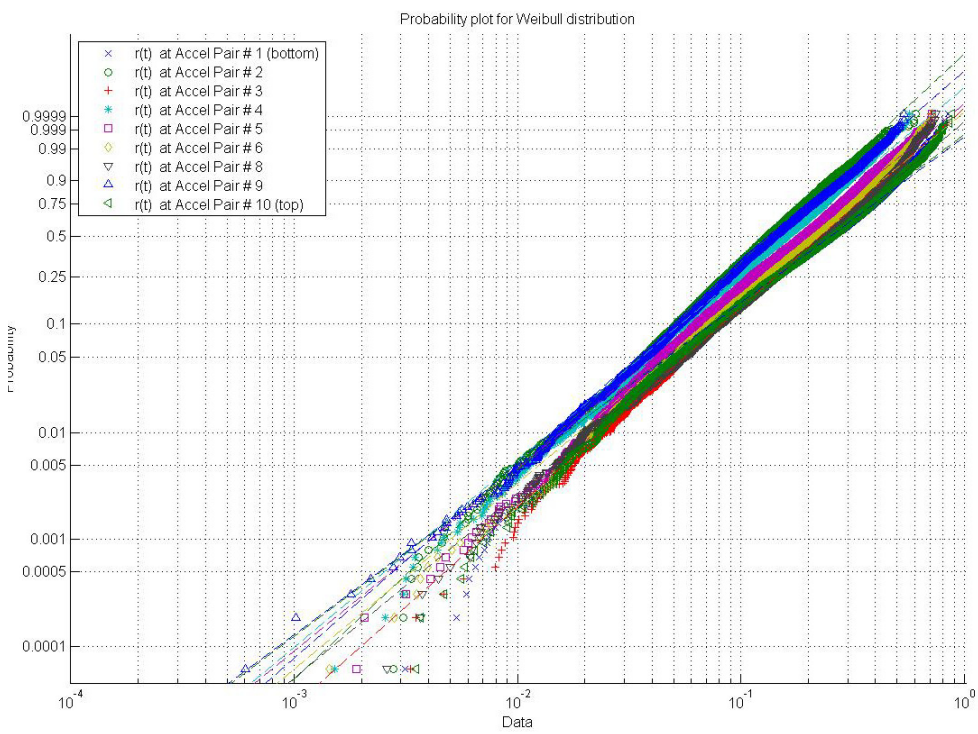

(b)

Figure 4: Weibull probability plots of the radial displacement at each working bi-axial accelerometer (a) complete measurement and, (b) the truncated records. 


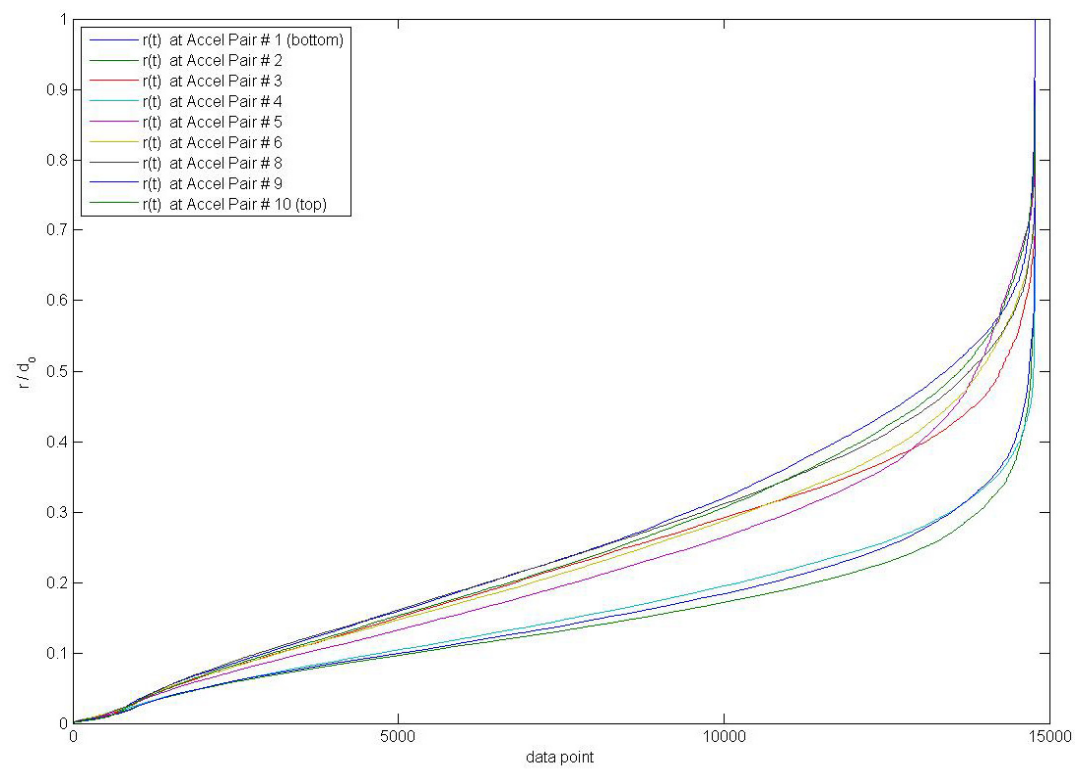

(a)

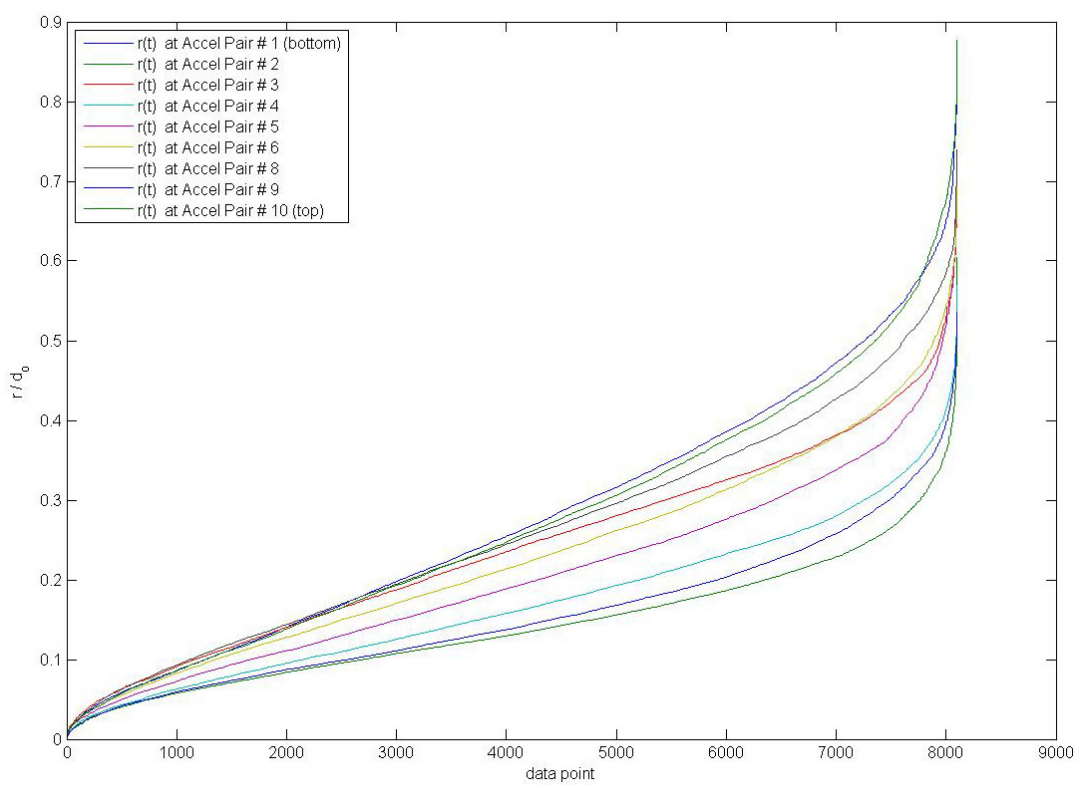

(b)

Figure 5: Comparison sorted radial displacement at each working bi-axial accelerometer (a) complete measurement and, (b) the truncated records. 

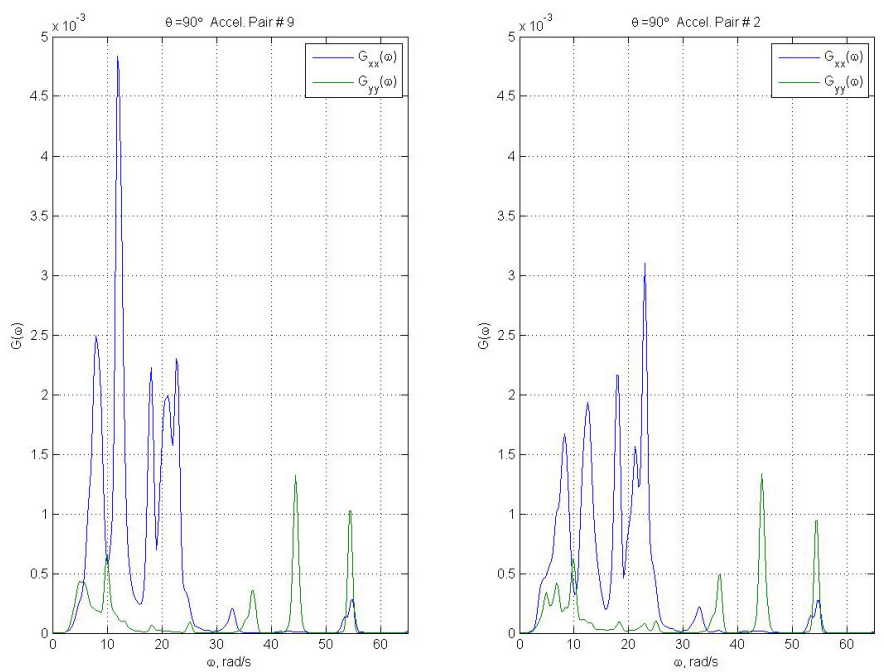

(a)
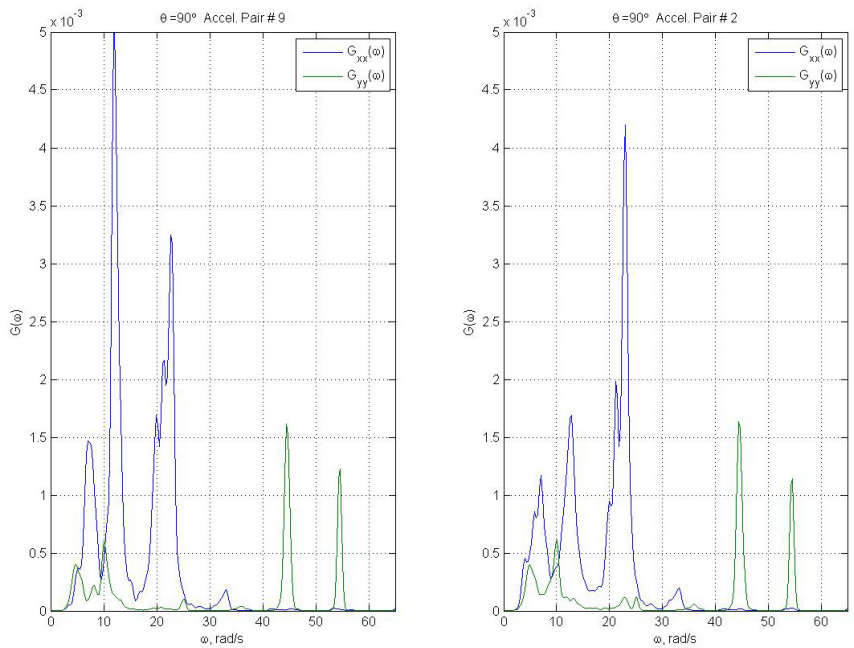

(b)

Figure 6: Comparison of auto-spectral estimates at each working bi-axial accelerometer (a) complete measurement and, (b) truncated records.

\section{Closure}

The VIV response behaviour of a SCR model towed to simulate uniform current flow conditions was studied in an effort to gain insight into the nature of the 
vortex induced motions during the start-up and ending of the test run. The influence of time series truncation based upon physical reasoning using the time series was investigated. A transformation of the displacement components to Polar coordinates was introduced to focus on the radial amplitudes of the vortex induced motion. In the analysis it was assumed that the catenary vibration modes did not have a significant vertical motion at each measurement location along the SCR model. The effects of truncation on the analysis of the records was investigated and shown to have an influence that was detectable in each of the graphs presented. The peak frequencies of the multi-mode response remained fairly consistent, and the peak amplitudes were somewhat different. Interestingly, estimates of the peak frequencies of VIV were quite constituent with those predicted by accounting for both the axial and bending stiffness.

\section{Acknowledgements}

The authors would like to express their appreciation for the permission to use the STRIDE experimental data granted by $2 \mathrm{H}$ Offshore Engineering Ltd. and the project sponsors. A special expression of appreciation is also extended to Halvor Lie of MARINTEK, Norway. The first writer would also like to acknowledge the partial support of the R.P. Gregory '32 Chair during this research study.

\section{References}

[1] Teigen, T., VIV of a SCR due to current from any direction, Department of Structural Engineering, Thesis, NTNU, Trondheim Norway, June 2002.

[2] Moe, G., Teigen, T. \& Lie, H. Predictions and model tests of a SCR undergoing VIV in flow at oblique angles, OMAE 51563, pp. 12, 2004.

[3] Larsen, C.M., Workshop on vortex induced vibrations, Centre of Excellence for Ships and Ocean Structures, NTNU, Trondheim Norway, October 2004.

[4] Mercier, R.S., Workshop on vortex induced motion of ocean structures, Offshore Technology Research Center, Texas A\&M University, Oct. 2003.

[5] Niedzwecki, J.M. \& Moe, G., Investigation of a catenary riser undergoing VIV, WIT Conference Fluid Structure Interaction 2005, pp. 8, 2005 